\title{
Monte Carlo simulation method for Laughlin-like states in a disk geometry
}

\author{
Orion Ciftja and Carlos Wexler \\ Department of Physics and Astronomy, University of Missouri-Columbia, Columbia, Missouri 65211 \\ (Received 22 May 2002; revised manuscript received 20 August 2002; published 6 February 2003)
}

\begin{abstract}
We discuss an alternative accurate Monte Carlo method to calculate the ground-state energy and related quantities for Laughlin states of the fractional quantum Hall effect in a disk geometry. This alternative approach allows us to obtain accurate bulk regime (thermodynamic limit) values for various quantities from Monte Carlo simulations with a small number of particles (much smaller than that needed with standard Monte Carlo approaches).
\end{abstract}

DOI: 10.1103/PhysRevB.67.075304

PACS number(s): 73.43.-f, 05.30.Fk, 71.70.Di

\section{INTRODUCTION}

The discovery of the fractional quantum Hall effect (FQHE) has stimulated extensive studies on the properties of two-dimensional (2D) quantum many-electron systems in a strong magnetic field. ${ }^{1}$ It is now understood that the FQHE represents the condensation of nearly $2 \mathrm{D}$ electrons subject to a strong perpendicular magnetic field (at low enough temperatures and low enough amount of disorder) into an incompressible quantum fluid formed at some specific filling factors. A neutralizing positive charge density is present to preserve overall charge neutrality and, to lowest approximation, can be thought of as a uniform positive density in the same plane as the 2D electrons (in reality the positive charges are the ionized donors that are roughly distributed randomly a spacer thickness away and produce a small amount of disorder and an overall constant shift in the energies).

The strong magnetic field quantizes the electrons's motion on the plane and quenches the kinetic energy of each electron to a discrete set of Landau levels (LL's) separated by the relatively large cyclotron energy $\hbar \omega_{c}=\hbar e B / m^{*}$, where $-e(e>0)$ is the electron charge and $m^{*}$ is the effective mass of electrons in the semiconductor $\left(m^{*} \simeq 0.07 m_{e}\right.$ in GaAs, where $m_{e}$ is the bare electron's mass). In addition, the Zeeman splitting spin-polarizes the electrons rendering them effectively spinless. In each LL, $\rho_{L L}=1 / 2 \pi l_{0}^{2}\left(l_{0}=\sqrt{\hbar / e B}\right.$ is the magnetic length) electrons per unit area can be accommodated. It is evident that for large enough magnetic fields only the lowest LL (LLL) is occupied (and only the lowest spin sub-band), and if the occupation is not complete the system is highly degenerate. At particular filling factors $\nu$ $=\rho / \rho_{L L}$, electron interactions lead to highly correlated states which exhibit an excitation gap and result in the observed FQHE. These particular filling factors form a hierarchy, of which the simplest FQHE states have filling factors $\nu=1 / m$ with $m=3$ and 5 .

For filling factors of the form $\nu=1 / m$ (m odd) the unnormalized Laughlin ${ }^{2}$ trial wave function for $N$ electrons can be written as

$$
\Psi_{m}\left(z_{1} \ldots z_{N}\right)=\prod_{i<j}^{N}\left(z_{i}-z_{j}\right)^{m} \prod_{i=1}^{N} e^{-\left|z_{i}\right|^{2} / 4 l_{0}^{2}},
$$

where $z_{j}=x_{j}+i y_{j}$ is the position of the $j$ th electron in complex coordinates. This wave function gives an excellent description of the true ground state of the electrons for $m=3$ and 5. For $m \geqslant 7$ the electrons tend to form a Wigner crystal ${ }^{3}$ consistent with the experimental observation ${ }^{4}$ that the FQHE does not occur for filling factors $\nu \leqslant 1 / 7$.

Since the Laughlin wave function lies entirely in the LLL, the expectation value of the kinetic energy per electron,

$$
\frac{\langle\hat{K}\rangle}{N}=\frac{1}{2} \hbar \omega_{c},
$$

is constant and becomes irrelevant, therefore the only important contribution of the quantum-mechanical Hamiltonian $\hat{H}=\hat{K}+\hat{V}$ originates from the total potential-energy operator:

$$
\hat{V}=\hat{V}_{e e}+\hat{V}_{e b}+\hat{V}_{b b},
$$

where

$$
\begin{gathered}
\hat{V}_{e e}=\sum_{i<j}^{N} \frac{e^{2}}{\left|\mathbf{r}_{i}-\mathbf{r}_{j}\right|} ; \quad \hat{V}_{e b}=-\rho_{0} \sum_{i=1}^{N} \int_{\Omega_{N}} d^{2} r \frac{e^{2}}{\left|\mathbf{r}_{i}-\mathbf{r}\right|} ; \\
\hat{V}_{b b}=\frac{\rho_{0}^{2}}{2} \int_{\Omega_{N}} d^{2} r \int_{\Omega_{N}} d^{2} r^{\prime} \frac{e^{2}}{\left|\mathbf{r}-\mathbf{r}^{\prime}\right|}
\end{gathered}
$$

are the electron-electron, electron-background, and background-background potential-energy operators, respectively. Here we have assumed a simple geometry appropriate for the circular symmetry of the Laughlin wave function, where a positive background density $\rho_{0}=\nu /\left(2 \pi l_{0}^{2}\right)$ is spread over a disk $\Omega_{N}$ of radius $R_{N}=l_{0} \sqrt{2 N / \nu}$ (i.e., it cancels the electronic density in the thermodynamic limit and makes the system neutral for all $N$ ).

Numerous techniques have been employed to calculate the expectation value of the potential energy per particle $\langle\hat{V}\rangle / N$ [see Eq. (3)] in the Laughlin state [Eq. (1)]. For example, Laughlin ${ }^{2}$ initially employed the hypernetted-chain method to estimate the value of this correlation energy with a $\sim 1 \%$ accuracy); and various standard Monte Carlo (MC) schemes have been proposed, all of which are essentially exact in the thermodynamic limit.

An excellent description of a standard MC computation of the potential energy and other relevant quantities in a disk geometry is given by Morf and Halperin. ${ }^{5}$ Spherical geom- 
etries are also used quite often since the convergence to the thermodynamic limit is quicker because boundary effects are eliminated. ${ }^{6}$

Although considerable more care is needed in the disk geometry to eliminate boundary effects when extrapolating (necessarily) finite- $N$ results to the thermodynamic limit (in particular due to the long-range nature of the Coulomb potential), there are cases in which the spherical geometry is either inconvenient, or plainly incompatible with the state under consideration (for example for the study of possible quantum Hall nematic phases ${ }^{7}$ for which considerable topological defects would be generated at the poles of the sphere).

Furthermore, the value of the correlation energy in the thermodynamic limit is not easily extracted from standard MC simulation (see Sec. II) data, since the limit is approached very slowly, with corrections of the order of $1 / \sqrt{N}$, requiring repeated calculations for various $N$ and a careful extrapolation of the results to the $N \rightarrow \infty$ limit. It is therefore highly desirable to explore methods that would expedite the extrapolation to the thermodynamic limit.

In Sec. II we describe, for the sake of completeness, the procedure used in the standard MC approach. Section III describes an alternative method that converges to the thermodynamic limit considerably faster. We discuss our results in Sec. IV.

\section{STANDARD MONTE CARLO APPROACH}

In the standard MC approach one considers the calculation of the expectation value of the potential-energy operators as given in Eq. (4). The background-background interaction potential poses no problem, it can be calculated analytically and is found to be

$$
\frac{\left\langle\hat{V}_{b b}\right\rangle}{N}=\frac{1}{N} \frac{\rho_{0}^{2}}{2} \int_{\Omega_{N}} d^{2} r \int_{\Omega_{N}} d^{2} r^{\prime} \frac{e^{2}}{\left|\vec{r}-\vec{r}^{\prime}\right|}=\frac{8}{3 \pi} \sqrt{\frac{\nu N}{2}} \frac{e^{2}}{l_{0}} .
$$

In order to compute the expectation value of the electronbackground interaction potential one conveniently writes it as

$$
\hat{V}_{e b}=\sum_{i=1}^{N} \hat{v}_{e b}\left(\mathbf{r}_{i}\right) ; \quad \hat{v}_{e b}\left(\mathbf{r}_{i}\right)=-\rho_{0} \int_{\Omega_{N}} d^{2} r \frac{e^{2}}{\left|\mathbf{r}_{i}-\mathbf{r}\right|},
$$

where $\hat{v}_{e b}\left(\mathbf{r}_{i}\right)$ is the interaction potential of a single electron at position $\vec{r}_{i}$ with the uniform positive background in the finite disk. Such electron-background interaction potential depends on the ratio $r_{i} / R_{N}$, where $r_{i}=\left|\mathbf{r}_{i}\right|$ is electron's distance from the center of the disk and $R_{N}$ is the radius of the finite disk and can be expressed as

$$
\hat{v}_{e b}\left(\mathbf{r}_{i}\right)=-\sqrt{2 \nu N} F\left(r_{i} / R_{N}\right) \frac{e^{2}}{l_{0}}
$$

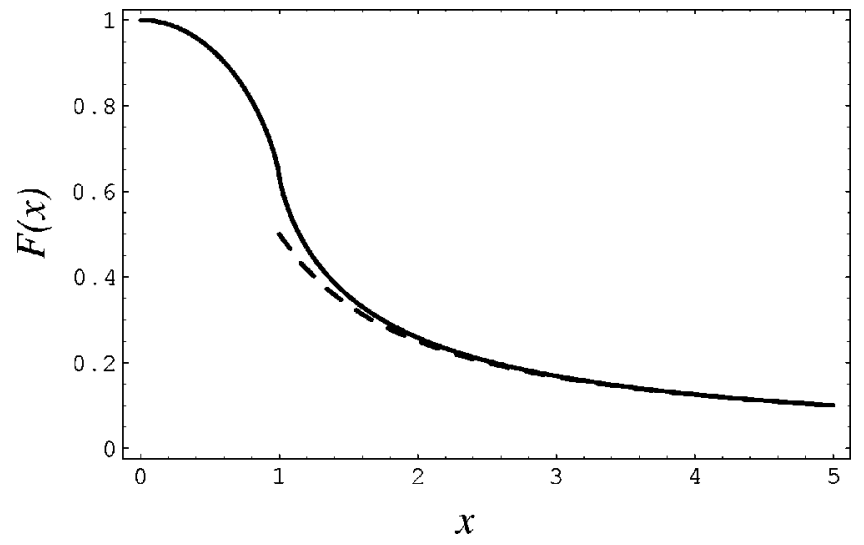

FIG. 1. The electron-background function $F(x)$. Here $x$ $=r / R_{N}$, where $r$ is the distance of an electron from the center of the disk and $R_{N}$ is the radius of the finite disk filled with neutralizing positive background. The dotted line shows the $1 /(2 x)$ asymptotic dependence. Most electrons sit in the $x \leqslant 1$ region.

$$
F(x)=\int_{0}^{\infty} d y \frac{J_{0}(x y) J_{1}(y)}{y}=\left\{\begin{array}{l}
\frac{2 E\left(x^{2}\right)}{\pi}, \quad x \leqslant 1 \\
\frac{{ }_{2} F_{1}\left(\frac{1}{2}, \frac{1}{2} ; 2 ; \frac{1}{x^{2}}\right)}{2 x}, \quad x \geqslant 1,
\end{array}\right.
$$

where $J_{n}(x)$ are Bessel functions of order $n, E(x)$ is the complete elliptic integral, and ${ }_{2} F_{1}(a, b ; c ; z)$ is the hypergeometric function. Figure 1 plots the function $F(x)$. It is interesting to note that $F(0)=1, F(1)=2 / \pi$, and $F(x)$ $\sim 1 /(2 x)$ for $x \gg 1$, as expected for the Coulomb potential far from a charged disk. Although $F(x)$ can be expressed analytically, it generally preferable to store it in a table, and interpolate it for all $x$ for all the calculations that follow.

While most electrons stay within the confines of the neutralizing background (i.e., $x \leqslant 1$ ), electrons near the edge may spread outside the disk to some extent (although it is extremely unlikely that they will spread to more than a few magnetic lengths from the edge). The expectation value of the electron-background interaction potential during the MC simulation can then be calculated using

$$
\frac{\left\langle\hat{V}_{e b}\right\rangle}{N}=\frac{1}{N}\left\langle\sum_{i=1}^{N} \hat{v}_{e b}\left(\mathbf{r}_{i}\right)\right\rangle .
$$

Finally, the expectation value of $\hat{V}_{e e}$ is accordingly given by

$$
\frac{\left\langle\hat{V}_{e e}\right\rangle}{N}=\frac{1}{N}\left\langle\sum_{i<j}^{N} \frac{e^{2}}{\left|\mathbf{r}_{i}-\mathbf{r}_{j}\right|}\right\rangle .
$$

In the usual Metropolis MC method, ${ }^{8}$ the expectation value of an operator can be computed by averaging the value of the operator over numerous configurations $\left\{\vec{r}_{1}, \ldots, \vec{r}_{N}\right\}$ of the many-body system that obey detailed balance, that is, the probability ratios between pairs of discrete configurations are related by the ratios of the probability distribution for the 
TABLE I. Correlation energy per particle in the Laughlin state for filling factors $\nu=1 / 3$ and $1 / 5$. These results were obtained after a standard Monte Carlo simulation in a disk geometry. Energies are in units of $e^{2} / l_{0}$.

\begin{tabular}{lcc}
\hline \hline$N$ & $m=3$ & $m=5$ \\
\hline 4 & -0.38884 & -0.32159 \\
16 & -0.39766 & -0.32328 \\
36 & -0.40129 & -0.32446 \\
64 & -0.40323 & -0.32510 \\
100 & -0.40445 & -0.32550 \\
144 & -0.40521 & -0.32577 \\
196 & -0.40579 & -0.32594 \\
400 & -0.40675 & -0.32624 \\
\hline \hline
\end{tabular}

system [in this case $\left|\Psi_{m}\left(z_{1}, \ldots, z_{N}\right)\right|^{2}$; see Eq. (1)]. Usually several million configurations are used for each $N$ and the results are extrapolated to the thermodynamic limit by considering a sequence of various increasing $N$ 's.

A MC step (MCS) consists of attempts to move one by one all the electrons of the system by a small distance of order $\Delta$ in a random direction. After each attempt (to move the $i$ th electron from $\vec{r}_{i}^{\text {old }}$ to $\vec{r}_{i}^{\text {new }}$, the probability ratio between the "new" state and the "old" state is then computed:

$$
\begin{aligned}
\frac{\left|\Psi\left(\mathbf{r}_{1}, \cdots \mathbf{r}_{i}^{\text {new }} \cdots \mathbf{r}_{N}\right)\right|^{2}}{\left|\Psi\left(\mathbf{r}_{1}, \cdots \mathbf{r}_{i}^{\text {old }} \cdots \mathbf{r}_{N}\right)\right|^{2}}= & \exp \left[m \sum _ { j \neq i } ^ { N } \left(\ln \left|\mathbf{r}_{j}-\mathbf{r}_{i}^{\text {new }}\right|^{2}\right.\right. \\
& \left.\left.-\ln \left|\mathbf{r}_{j}-\mathbf{r}_{i}^{\text {old }}\right|^{2}\right)\right] \cdot \exp [ \\
& \left.-\frac{1}{2 l_{0}^{2}}\left(\left|\mathbf{r}_{i}^{\text {new }}\right|^{2}-\left|\mathbf{r}_{i}^{\text {old }}\right|^{2}\right)\right] .
\end{aligned}
$$

In the usual Metropolis scheme, ${ }^{8}$ if this ratio is bigger than a uniformly distributed number in the $[0,1]$ range the attempt is accepted, otherwise it is rejected. The parameter $\Delta$ is adjusted so that the acceptance ratio is close to $50 \%$. After attempting to move all electrons (one MCS), the electron configurations are then used to calculate the operator under consideration. Averaging over numerous MCS's converges gradually (as $1 / \sqrt{\text { number of MCS }}$ ) to the desired expectation value. Normally it is convenient to disregard numerous (several thousand) initial configurations to reach a good "thermalization" before the averaging begins, which significantly reduces the expurious effects of the somewhat arbitrary initial configurations. All the results that we report here were obtained after discarding 100000 "thermalization" MCS's and using $2 \times 10^{6}$ MCS's for averaging purposes.

In Table I we show the correlation energy per particle for finite systems of $N$ electrons in the Laughlin states $m=3$ and $m=5$ obtained using the standard MC method described above. The results are rounded in the last digit.

To get the the bulk (thermodynamic estimate) of the correlation energy per particle one needs to perform a careful extrapolation of the results as illustrated in Fig. 2 where we show the correlation energy per particle for states $\nu=1 / 3$ and
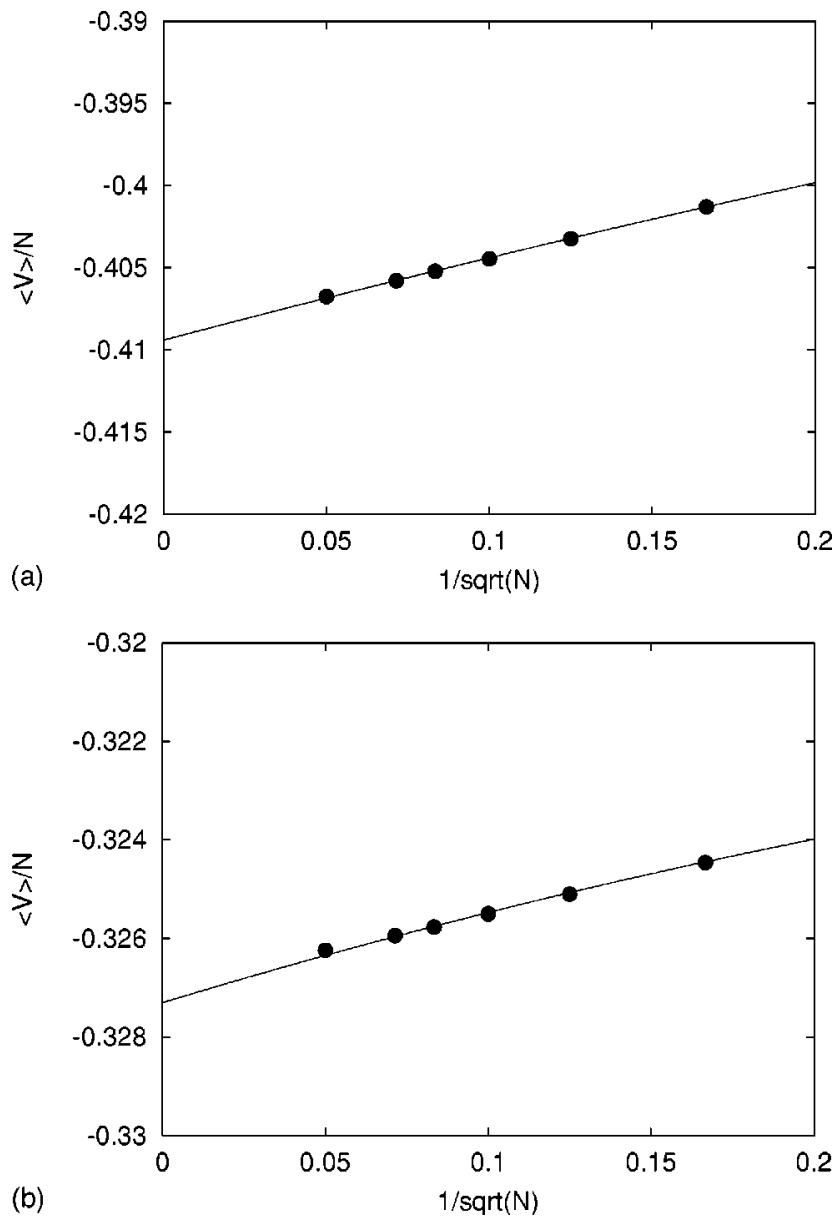

FIG. 2. Monte Carlo results in disk geometry for the Laughlin state at $\nu=1 / 3$ (top panel) and $\nu=1 / 5$ (bottom panel). The potential energy per particle, $\langle\hat{V}\rangle / N$, is plotted as a function of $1 / \sqrt{N}$ for systems with $N=36,64,100,144,196$, and 400 electrons. Full circles: correlation energies calculated by the standard method described above, the full line is a least-square fit [Eqs. (11) and (12)] used to extrapolate to the thermodynamic limit. Energies are in units of $e^{2} / l_{0}$.

$1 / 5$ plotted as a function of $1 / \sqrt{N}$ for systems with $N=36$, 64, 100, 144, 196, and 400 electrons.

We fitted the energies of Table I for $N=4,16,36,64$, $100,144,196$, and 400 electrons to a polynomial function as reported in Ref. 5 and obtained

$$
\begin{aligned}
& \frac{\langle\hat{V}\rangle_{1 / 3}}{N}=\left(-0.4094+\frac{0.0524}{\sqrt{N}}-\frac{0.0225}{N}\right) \frac{e^{2}}{l_{0}}, \\
& \frac{\langle\hat{V}\rangle_{1 / 5}}{N}=\left(-0.3273+\frac{0.0200}{\sqrt{N}}-\frac{0.0172}{N}\right) \frac{e^{2}}{l_{0}} .
\end{aligned}
$$

These interpolation lines are used to estimate the correlation energy per particle in the thermodynamic limit (the first term in each of the parentheses). Our results for the thermodynamic limit are similar to those found in Ref. 9, -0.4100 \pm 0.0001 and $-0.3277 \pm 0.0002$ (in units of $e^{2} / l_{0}$ ) derived with the use of the pair-correlation function evaluated from 


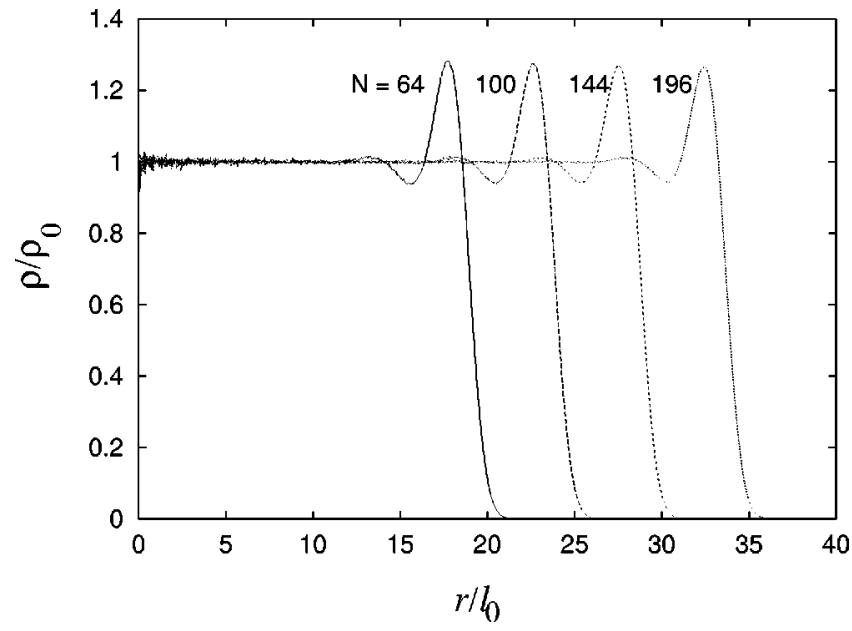

FIG. 3. One-body density function, $\rho(r) / \rho_{0}$, in the Laughlin state $\nu=1 / 3$ as a function of the distance $r / l_{0}$ from the center of the disk for systems with $N=64,100,144$, and 196 electrons. Note the persistence of an "edge region" of finite width and the development of a "bulk region" for large $N$. A discretization interval $\Delta r$ $=0.05 l_{0}$ was used.

MC simulations with up to $N=256$ electrons and generating as many as five million MC configuratons. Note how slow the convergence is, although the extrapolation to $N \rightarrow \infty$ seems unambiguous it is still time consuming and cumbersome (even for $N=400$ the error is still approximately $1 \%$ ).

\section{A. One-particle density}

Other physical quantities of interest that may be readily computed are the single-particle density function and the pair distribution function. Given that the Laughlin wave function describes an isotropic liquid and is rotationally invariant, the single-particle density depends only on the radial distance from the center of the disk. We may compute the singleparticle density by counting the number of electrons $N_{l}(\Delta r)$ found in several 2D shells of width $\Delta r$ centered around a discrete set of distances to the center $r_{l}=\left(l+\frac{1}{2}\right) \Delta r(l=0,1$, $\cdots)$ :

$$
\rho\left(r_{l}\right) \equiv\left\langle\frac{N_{l}(\Delta r)}{\Omega_{l}(\Delta r)}\right\rangle,
$$

where $\Omega_{l}(\Delta r)=\pi(\Delta r)^{2}\left[(l+1)^{2}-l^{2}\right]$ is the area of each 2D shell. In the $\Delta r \rightarrow 0$ the quantity computed corresponds unequivocally to the electron density

$$
\rho(r)=\left\langle\sum_{i=1}^{N} \delta\left(r-r_{i}\right)\right\rangle
$$

The computation of the single-particle density in the Laughlin state indicates a significant nonuniformity near the boundary (see Fig. 3). As the number of electrons increase, a significant portion of the system becomes uniform as expected. Note, however, that the nonuniformity near the edge always persists. This behavior can be used to characterize which electrons are "in the bulk."

\section{B. Pair distribution function}

Another important quantity related with the trial wave function is the pair distribution function, which corresponds to the conditional probability density to find an electron at a distance $r$ from another electron. For any homogeneous and isotropic liquid with uniform density $\rho_{0}$ it is defined as

$$
\rho_{0} g(r)=\frac{1}{N}\left\langle\sum_{i=1}^{N} \sum_{j \neq i}^{N} \delta\left(r-\left|\mathbf{r}_{i}-\mathbf{r}_{j}\right|\right)\right\rangle .
$$

Following the same procedure as above, we discretize in concentric shells around the $i$ th electron and count the number of electrons $N_{l}(\Delta r)$ in each shell, which should give $g(r)$ as $\Delta r \rightarrow 0$ according to the following equation:

$$
\rho_{0} g\left(r_{l}\right)=\frac{1}{N} \frac{1}{\Omega_{l}(\Delta r)}\left\langle\sum_{i=1}^{N} \sum_{j \neq i}^{N} N_{l}(\Delta r)\right\rangle .
$$

It is evident that electrons near the edges of the system could contribute expuriously to these sums as their "surroundings" are considerably different than those at the bulk. To eliminate as much as possible any boundary effects, it is convenient ${ }^{5}$ to consider only (for the " $i$ electrons" above) the electrons that are within a small circle of radius $R_{1}$ around the origin. If $N_{1}$ is the average number of electrons that are within this small circle, then the approximation $\hat{g}\left(r_{l}\right)$ for the pair distribution $g\left(r_{l}\right)$ is

$$
\rho_{0} \hat{g}\left(r_{l}\right)=\frac{1}{N_{1}} \frac{1}{\Omega_{l}(\Delta r)}\left\langle\sum_{i=1}^{N_{1}} \sum_{j \neq i}^{N} N_{l}(\Delta r)\right\rangle,
$$

where in this expression one is considering the pairs between any electron $i\left(i=1, \ldots, N_{1}\right)$ lying inside the circle of radius $R_{1}$, with all other electrons $j(j=1, \ldots, i-1, i+1, \ldots, N)$ that may lie either inside, or outside that circle. This guarantees that the evaluation of $\hat{g}\left(r_{l}\right)$ involves only pairs, where at least one member lies inside a circle of radius $R_{1}$ around the origin, where correlations are believed to be close to those in the bulk of an infinite system.

Figure 4 show plots of the pair distribution function for the states $\nu=1 / 3$ and $1 / 5$ for systems with $N=4,16,36,64$, 100, 144, and 196 electrons. For our MC simulations we chose $R_{1}=0.25 R_{N}$ and a discretization interval $\Delta r=0.05 l_{0}$. Note the gradual decay at large $r$ which reflects the finite size of the system.

The determination of the pair correlation function for a given finite $N$ tends to be quite time consuming but provides for an alternative way to compute the correlation energy per particle in the thermodynamic $\operatorname{limit}^{5}$ by using the formula

$$
\frac{\langle\hat{V}\rangle}{N}=\frac{\rho_{0}}{2} \int_{0}^{+\infty} d^{2} r \frac{e^{2}}{r}[g(r)-1],
$$

which is valid in the limit of an infinite system. Although the pair distribution function is obtained from a system with a finite number of particles, one can calculate the thermodynamic value of the correlation energy per particle to a very good accuracy by using the slightly modified formula 

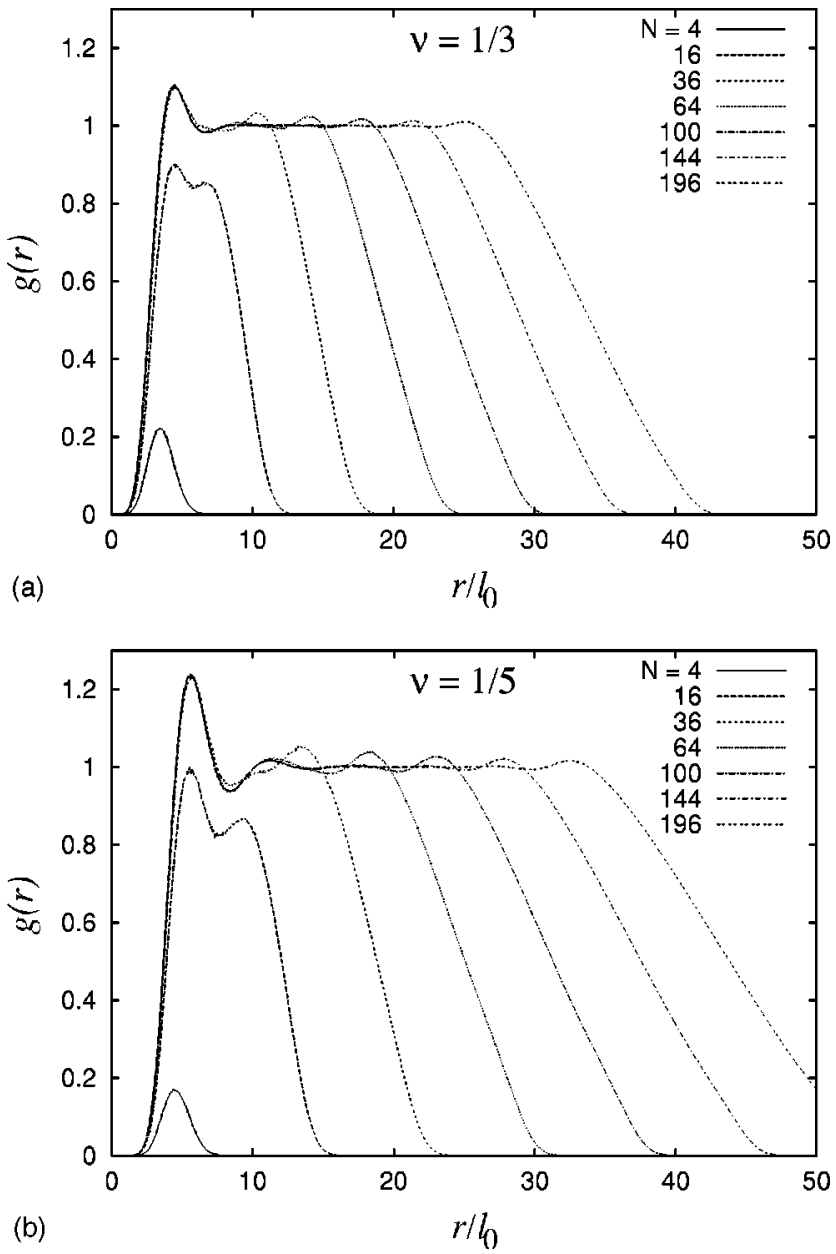

FIG. 4. Pair distribution function for $\nu=1 / 3$ and $1 / 5$ obtained by a standard Monte Carlo simulation in disk geometry for systems of $N=4,16,36,64,100,144$, and 196 electrons.

$$
\frac{\langle\hat{V}\rangle}{N}=\frac{\rho_{0}}{2} \int_{0}^{R_{\text {cut }}} d^{2} r \frac{e^{2}}{r}[\hat{g}(r)-1],
$$

in conjunction with the normalization condition

$$
\rho_{0} \int_{0}^{R_{c u t}} d^{2} r[\hat{g}(r)-1]=-1,
$$

which defines an upper cuttoff $R_{c u t}$. This approach produces good estimates for the thermodynamic correlation energy per particle as long as $\hat{g}(r)$ is able to reach its asymptotic value $[\hat{g}(r) \simeq 1]$. Reasonable results can be achieved even for systems of $N \geqslant 36$ electrons.

\section{AN ALTERNATIVE METHOD}

In the standard MC approach one needs to calculate the expectation values of various quantities several times for different $N$ in order to extract the thermodynamic estimate from the data by performing a $1 / \sqrt{N}$ fit (and taking the limit $1 / \sqrt{N} \rightarrow 0$, as shown, e.g., in Fig. 2). It is highly desirable to obtain estimates for the bulk regime (in thermodynamic limit) without needing to perform the above analysis. In the following we describe a method that allows us to obtain results consistent with the bulk regime, by doing simulations with only a finite (relatively small) number of particles. The method although approximate, yields very accurate results corresponding to the bulk regime even when simulations are performed for a small number of electrons. The estimates are very stable over a wide range of $N$ and the technical application of the simulation is less involved.

In order to obtain reliable estimates for the bulk regime, we need to exclude from consideration the boundary-affected outer region of the finite disk. In a standard MC simulation the electrons are distributed all over the 2D space, at a given instant it is obvious that the electrons close to the central region of the disk resemble to the bulk regime better than those close to the boundary. However, during the simulation each of the previously "bulk" electrons moves around the whole disk therefore the correlation energy of such electron with the other electrons is not a good estimate of the correlation energy in the bulk regime.

The core of the method proposed here is to consider an electron pinned to the center of the disk which, by construction is the point which most closely resembles the bulk of the system. Therefore, if we are able to derive results where only the correlation energy of that particular electron with the rest (away from edge) is involved, we anticipate that such estimates should approximate the bulk regime much more accurately than other methods, and as a result thermodynamic limit values can be achieved even in a system with a relatively small number of electrons. One has to recall that for a finite system, the Laughlin wave function describes an incompressible system of strongly correlated ellectrons with uniform density $\rho_{0}$ only in the bulk region (central part of the disk not very close to the boundary), while close to the boundary (where the density of electrons falls to zero) the fluid becomes compressible and there is a deviation of the electron density from its constant value in the bulk.

In our MC simulations, we consider a Laughlin-like state [Eq. (1)] in which one electron is pinned at the position $z_{0}$ (we consider $z_{0}=0$ ), and $N^{\prime}=N-1$ electrons are free to move (i.e., in a typical MC step):

$\Psi_{m}^{\prime}\left(z_{0}, z_{1}, \cdots z_{N^{\prime}}\right)=\Psi_{m}\left(z_{1}, \cdots z_{N^{\prime}}\right) \prod_{j=1}^{N^{\prime}}\left(z_{j}-z_{0}\right)^{m} e^{-\left|z_{0}\right|^{2} / 4 l_{0}^{2}}$.

When an attempt is made to move electron $i$ ( $i$ $\left.=1, \ldots, N^{\prime}\right)$ from $\mathbf{r}_{i}^{\text {old }}$ to $\mathbf{r}_{i}^{\text {new }}$, the MC probability ratio is given by

$$
\begin{aligned}
\frac{\left|\Psi^{\prime}\left(\mathbf{r}_{0}, \cdots \mathbf{r}_{i}^{\text {new }} \cdots \mathbf{r}_{N^{\prime}}\right)\right|^{2}}{\left|\Psi^{\prime}\left(\mathbf{r}_{0}, \cdots \mathbf{r}_{i}^{\text {old }} \cdots \mathbf{r}_{N^{\prime}}\right)\right|^{2}}= & \frac{\left|\Psi\left(\mathbf{r}_{1}, \cdots \mathbf{r}_{i}^{\text {new }} \cdots \mathbf{r}_{N^{\prime}}\right)\right|^{2}}{\left|\Psi\left(\mathbf{r}_{1}, \cdots \mathbf{r}_{i}^{\text {old }} \cdots \mathbf{r}_{N^{\prime}}\right)\right|^{2}} \\
& \times e^{m\left(\ln \left|\mathbf{r}_{i}^{\text {new }}-\mathbf{r}_{0}\right|^{2}-\ln \left|\mathbf{r}_{i}^{\text {old }}-\mathbf{r}_{0}\right|^{2}\right)} .
\end{aligned}
$$

Since the electrons are identical we need only consider the average correlation energy of one specified electron with the rest of the electrons to compute the electron-electron in- 
TABLE II. Correlation energy per particle in the Laughlin state for filling factors $\nu=1 / 3$ and $1 / 5$, obtained via a Monte Carlo simulation in a disk geometry using the method of pinning one electron at the center of the disk. Energies are in units of $e^{2} / l_{0}$.

\begin{tabular}{lcc}
\hline \hline$N$ & $m=3$ & $m=5$ \\
\hline 4 & -0.38187 & -0.30157 \\
16 & -0.40898 & -0.32722 \\
36 & -0.40895 & -0.32637 \\
64 & -0.40909 & -0.32665 \\
100 & -0.40955 & -0.32738 \\
144 & -0.40936 & -0.32732 \\
196 & -0.40953 & -0.32734 \\
400 & -0.40954 & -0.32735 \\
\hline \hline
\end{tabular}

teraction energy, therefore to this level of simplification the electron-electron energy per particle is obtained by considering only the interaction between the pinned electron and the rest of $N_{i}$ other electrons contained within a inner disk of radius $R_{i}<R_{N}$ where the electron density is approximately equal to the bulk value $\rho_{0}$. We found that a reasonable choice for the radius of inner disk that excludes the edge electrons is $R_{i}=0.75 R_{N}$, therefore this value was adopted in all the following simulations.

To be consistent with the above procedure also the disk's electron-background and background-background energy should be calculated within the same degree of simplification (see the Appendix). It is therefore useful to first calculate the one-body density function (see, e.g., Fig. 3) in order to determine an optimal $R_{i}$ for future use. If $R_{i}$ is reasonably large, we expect the correlation energy calculated in this way to closely correspond to the desired correlation energy per particle in the thermodynamic limit (see the Appendix):

$$
\frac{\langle\hat{V}\rangle}{N} \simeq \frac{1}{2}\left\langle\sum_{i=1}^{N_{i}} \frac{e^{2}}{\left|\vec{r}_{i}-\vec{r}_{0}\right|}\right\rangle-\sqrt{\frac{N_{i}+1}{2 m}} \frac{e^{2}}{l_{0}}
$$

As in the standard MC method case, our MC runs consist of 100000 discarded equalibration MCS's followed by $2 \times 10^{6}$ MCS's for averaging purposes.

In Table II we show the correlation energy per particle for finite systems of $N$ electrons and Laughlin states $m=3$ and $m=5$ calculated by pinning an electron at $z_{0}=0$ as described above [see Eq. (22)], and using Eq. (23). The results are rounded in the last digit.

In Fig. 5 we show the potential energy per particle for $\nu$ $=1 / 3$ and $1 / 5$ computed from the alternative method and plotted as a function of $1 / \sqrt{N}$. For the sake of comparison we also plot the results from the standard method (Sec. II).

It is striking to note how much faster the alternative method converges to the thermodynamic limit. Differently from the standard MC approach, the alternative method that we introduced does not need to have the data points leastsquare fitted to get the thermodynamic limit value. One merely needs to choose a big enough $N$ (for instance $N$
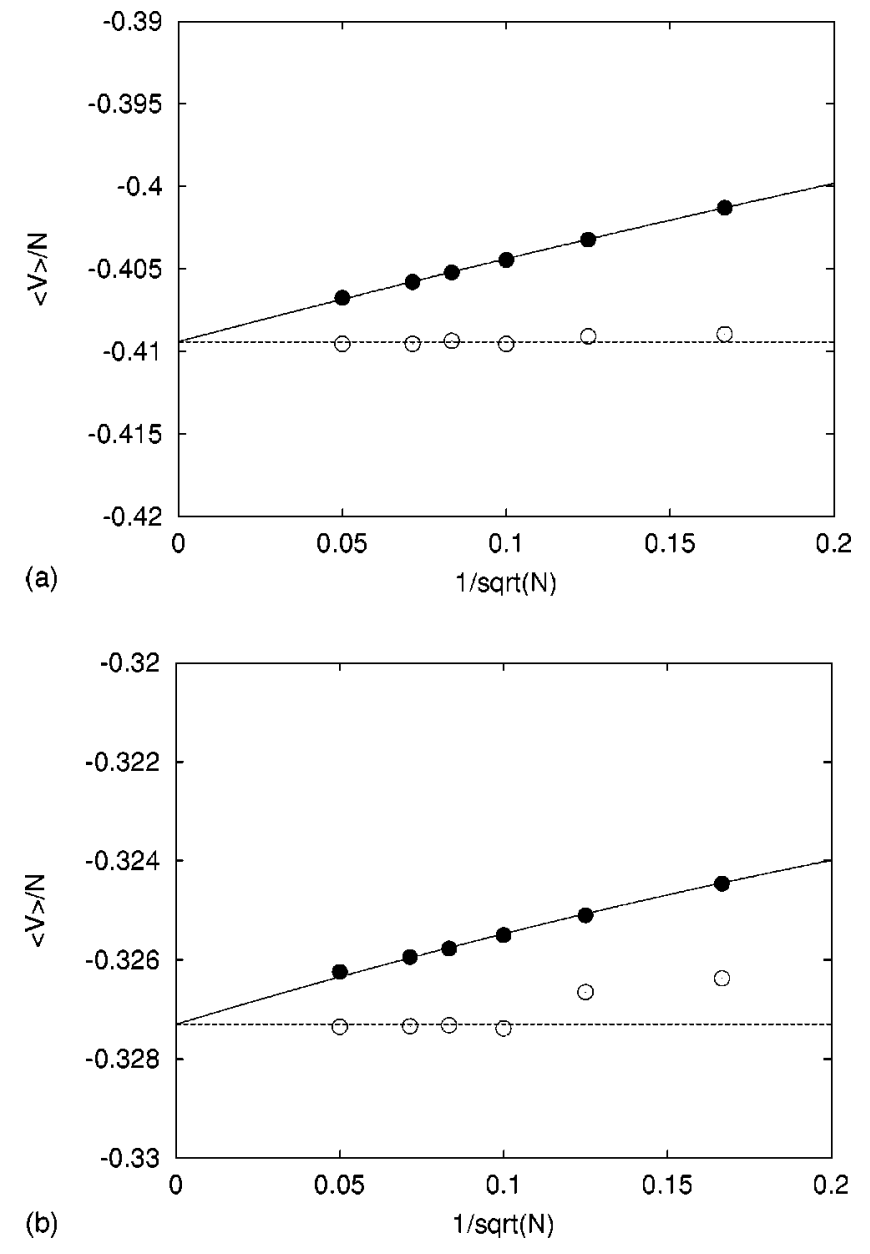

FIG. 5. Monte Carlo results in disk geometry for the Laughlin state at $\nu=1 / 3$ (top panel) and 1/5 (bottom panel). The potential energy per particle, $\langle\hat{V}\rangle / N$, is plotted as a function of $1 / \sqrt{N}$ for systems with $N=36,64,100,144,196$, and 400 electrons. Full circles: correlation energies calculated by the standard method (Sec. II) the full line is a least-square fit [Eqs. (11) and (12)] used to extrapolate to the thermodynamic limit. Empty circles: correlation energies calculated by the alternative method described in Sec. III, the dashed line is a visual aid indicating the thermodynamic limit. Note how the thermodynamic limit is approached faster in the second method. Energies are in units of $e^{2} / l_{0}$.

=196) and do a MC run that typically will generate bulk (thermodynamic limit) results to an excellent degree of accuracy.

\section{Pair distribution function}

Using the same ideas presented above (keep one electron pinned at $z_{0}=0$ ) the pair distribution function is very easily calculated. The essence of the method consists in measuring the one-particle density excluding the pinned electron which is obviously the pair distribution function instead of considering all the possible pairings between the electrons. As before, the advantage of this method, besides its simplicity, resides in the fact that this electron, being the farthest from the edge, is in an environment closest to that in a bulk system. Therefore one has to calculate 

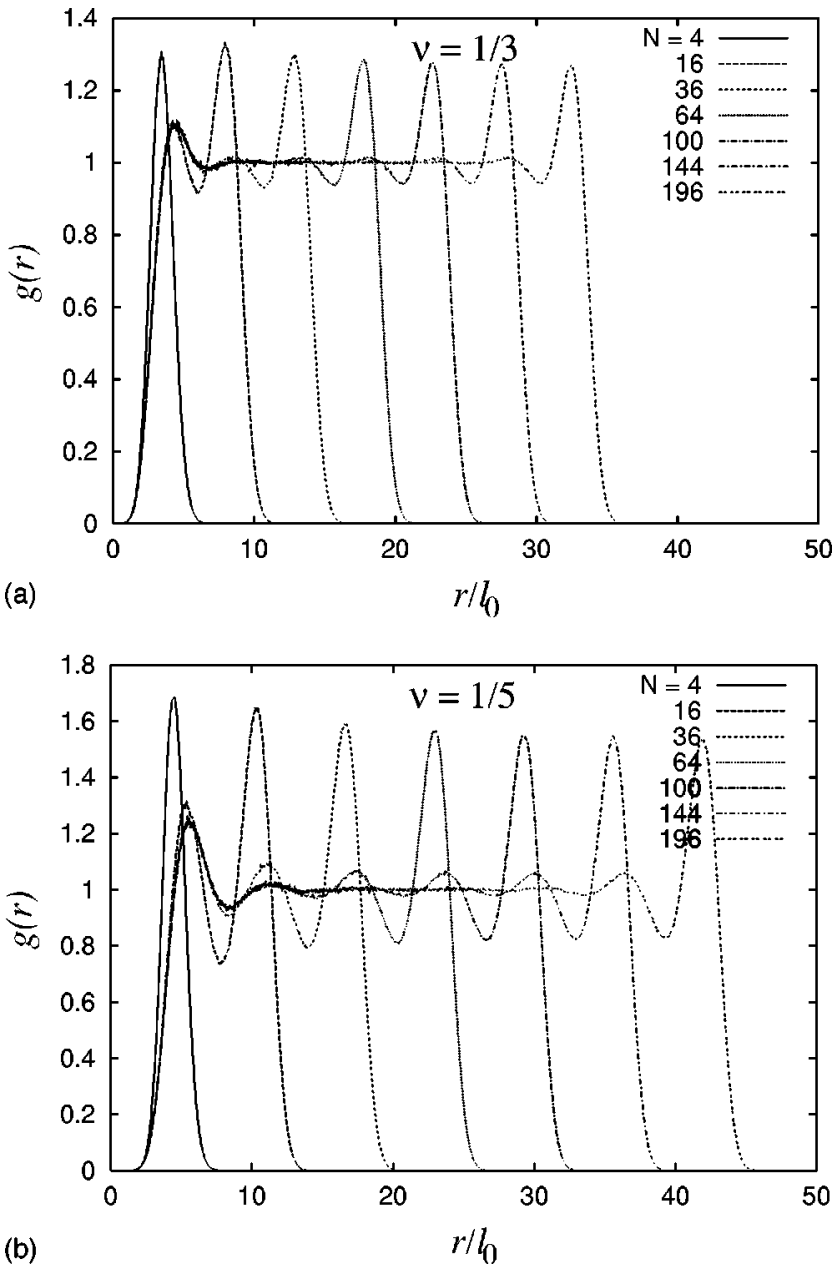

FIG. 6. Pair distribution function for the state $\nu=1 / 3$ and $1 / 5$ obtained after a Monte Carlo simulation in the disk geometry for systems of $N=4,16,36,64,100,144$, and 196 electrons with one electron held fixed at the center of the disk.

$$
\rho_{0} g(r) \simeq\left\langle\frac{N_{l}(\Delta r)}{\Omega_{l}(\Delta r)}\right\rangle,
$$

where $N_{l}(\Delta r)$ is the number of electrons found in the 2D shell $\Omega_{l}(\Delta r)$ with distance range $\left(r_{l}, r_{l}+\Delta r\right)$ from the pinned electron at the center of the disk. In Fig. 6 we show plots of the pair distribution function for the states $\nu=1 / 3$ and $\nu=1 / 5$ for systems with $N=4,16,36,64,100,144$, and 196 electrons obtained with a choice of $R_{i}=0.75 R_{N}$ and using a discretization interval $\Delta r=0.05 l_{0}$.

\section{SUMMARY AND DISCUSSIONS}

We implemented an alternative Monte Carlo method to calculate the properties of Laughlin states of the fractional quantum Hall effect in the thermodynamic limit while using a very small number of electrons. The key point of this method is the pinning of an electron in the center of the disk, so that the potential energy and correlation functions calculated through the pinned electron accurately represent the bulk (thermodynamic limit) even in a relatively small system. The idea is quite general and, in principle, can be ap- plied to any system as far as the main concern is the calculation of correlation effects such as the potential energy, the pair distribution function, etc. For systems such as 2D electronic one-component plasmas, ${ }^{10}$ composite fermion states described by the Jain's unprojected wave function, ${ }^{11}$ etc., that is all that matters. Obviously such alternative method can always be used to calculate the potential energy and related quantities of other more diverse systems, with the the kinetic energy calculated in the standard way whenever applicable. By using this alternative method we analyzed the properties of the Laughlin states corresponding to filling factor $\nu=1 / 3$ and $1 / 5$ by performing Monte Carlo simulations in disk geometry for systems with up to $N$ $=400$ electrons. The correlation energy per particle and the pair distribution function computed in this approach are compared to corresponding bulk-regime values obtained via a standard Monte Carlo simulation in disk geometry, where a careful extrapolation in thermodynamic limit has been performed. We find that such approach allows us to obtain accurate bulk regime (thermodynamic limit) values for various quantities using a modest number of electrons (even for $N$ $=16$ the error is less than $0.1 \%$ ).

\section{ACKNOWLEDGMENTS}

We would like to thank A. T. Dorsey and M. Fogler for useful discussions. Acknowledgment is made to the University of Missouri Research Board and to the Donors of the Petroleum Research Fund, administered by the American Chemical Society, for support of this research.

\section{APPENDIX}

Let us consider a system of $N$ interacting 2D electrons coupled to the positive neutralizing background that fills a finite disk and guarantees overall charge neutrality. The expectation value of the electron-background potential energy per particle can be written as

$$
\frac{\left\langle\hat{V}_{e b}\right\rangle}{N}=-\frac{\rho_{0}}{N} \int d^{2} r_{1} \rho\left(\mathbf{r}_{1}\right) \int_{\Omega_{N}} d^{2} r \frac{e^{2}}{\left|\mathbf{r}_{1}-\mathbf{r}\right|},
$$

where $\rho\left(\mathbf{r}_{1}\right)$ is the one-body (single) electron density function given by

$$
\begin{gathered}
\rho\left(\mathbf{r}_{1}\right)=N \frac{\int d^{2} r_{2} \cdots d^{2} r_{N}\left|\Psi\left(\mathbf{r}_{1} \cdots \mathbf{r}_{N}\right)\right|^{2}}{\int d^{2} r_{1} \cdots d^{2} r_{N}\left|\Psi\left(\mathbf{r}_{1} \cdots \mathbf{r}_{N}\right)\right|^{2}} \\
\int d^{2} r_{1} \rho\left(\mathbf{r}_{1}\right)=N .
\end{gathered}
$$

One notes that when the one-body electron density becomes uniform, $\rho\left(\mathbf{r}_{1}\right) \approx \rho_{0}$ and the system is sufficiently large so that most of the electrons are to be found inside the finite disk then $-\left\langle\hat{V}_{e b}\right\rangle / N \approx 2\left\langle\hat{V}_{b b}\right\rangle / N$, therefore we would have 


$$
\frac{\left\langle\hat{V}_{e b}\right\rangle+\left\langle\hat{V}_{b b}\right\rangle}{N} \simeq \frac{1}{2} \frac{\left\langle\hat{V}_{e b}\right\rangle}{N} .
$$

By making a preliminary calculation of the one-body density function we could estimate the radius, $R_{i}<R_{N}$ of an inner disk where the electrons have supposedly uniform density therefore we could argue that all these electrons inside this inner disk are in the bulk regime. If $N_{i}$ is the number of electrons within this reference circle of radius $R_{i}$ in addition to the electron pinned at the center of the disk then a good estimate for the potential energy per particle in the thermodynamic limit is obtained from the quantity

$$
\begin{aligned}
\frac{\langle\hat{V}\rangle}{N}= & \frac{1}{N_{i}+1}\left\langle\sum_{i=0}^{N_{i}} \sum_{i<j}^{N_{i}} \frac{e^{2}}{\left|\mathbf{r}_{i}-\mathbf{r}_{j}\right|}\right\rangle \\
& +\frac{1}{N_{i}+1}\left\langle\sum_{i=0}^{N_{i}} \hat{v}_{e b}\left(\mathbf{r}_{i}\right)\right\rangle+\frac{\left\langle\hat{V}_{b b}\right\rangle}{N_{i}+1},
\end{aligned}
$$

where $\left\langle\hat{V}_{b b}\right\rangle /\left(N_{i}+1\right)$ is the background-background energy per particle of a positive charge that exactly neutralizes the charge of $N_{i}+1$ electrons. Since the electron-electron poten- tial energy per particle was obtained to a level of simplification where only the interaction between the pinned electron and other $N_{i}$ electrons was considered, then the whole potential energy per particle should be calculated in the same grounds too, namely, using as reference only the interaction energy of the pinned electron with the positive background:

$$
\begin{aligned}
\frac{\langle\hat{V}\rangle}{N}= & \frac{\left\langle\hat{V}_{e e}\right\rangle}{N}+\frac{\left\langle\hat{V}_{e b}\right\rangle+\left\langle\hat{V}_{b b}\right\rangle}{N} \simeq \frac{1}{2}\left\langle\sum_{i=1}^{N_{i}} \frac{e^{2}}{\left|\vec{r}_{i}-\vec{r}_{0}\right|}\right\rangle \\
& +\frac{1}{2}\left\langle\hat{v}_{e b}\left(\mathbf{r}_{0}\right)\right\rangle=\frac{1}{2}\left\langle\sum_{i=1}^{N_{i}} \frac{e^{2}}{\left|\vec{r}_{i}-\vec{r}_{0}\right|}\right\rangle-\frac{\rho_{0}}{2} \int_{\Omega_{N_{i}}} d^{2} r \frac{e^{2}}{r}
\end{aligned}
$$

As a result the correlation energy per particle in the thermodynamic limit can be written as in Eq. (23). Note that use of relation $-\left\langle\hat{V}_{e b}\right\rangle / N \approx 2\left\langle\hat{V}_{b b}\right\rangle / N$ to express $\left(\left\langle\hat{V}_{e b}\right\rangle\right.$ $\left.+\left\langle\hat{V}_{b b}\right\rangle\right) / N$ in Eq. (A3) in terms of $\left\langle\hat{V}_{b b}\right\rangle / N$ is inaccurate in view of the approach adopted in our method therefore should be avoided.
${ }^{1}$ D. C. Tsui, H. L. Stormer, and A. C. Gossard, Phys. Rev. Lett. 48, 1559 (1982).

${ }^{2}$ R. B. Laughlin, Phys. Rev. Lett. 50, 1395 (1983).

${ }^{3}$ Pui K. Lam and S. M. Girvin, Phys. Rev. B 30, 473 (1984).

${ }^{4}$ E. Mendez, M. Heiblum, L. L. Chang, and L. Esaki, Phys. Rev. B 28, 4886 (1983).

${ }^{5}$ R. Morf and B. I. Halperin, Phys. Rev. B 33, 2221 (1986).

${ }^{6}$ R. Morf and B. I. Halperin, Z. Phys. B: Condens. Matter 68, 391 (1987).

${ }^{7}$ C. Wexler and O. Ciftja, J. Phys.: Condens. Matter 14, 3705
(2002); O. Ciftja and C. Wexler, Phys. Rev. B 65, 205307 (2002); 65, 045306 (2002).

${ }^{8}$ N. Metropolis, A. W. Rosenbluth, M. N. Rosenbluth, A. M. Teller, and E. Teller, J. Chem. Phys. 21, 1087 (1953).

${ }^{9}$ D. Levesque, J. J. Weis, and A. H. MacDonald, Phys. Rev. B 30, 1056 (1984).

${ }^{10}$ J. M. Caillol, D. Levesque, J. J. Weis, and J. P. Hansen, J. Stat. Phys. 28, 325 (1982).

${ }^{11}$ J. K. Jain, Phys. Rev. B 41, 7653 (1990). 